\title{
Critères et méthodologie d'évaluation au remboursement des anticancéreux
}

\author{
Rima de Sahb-Berkovitch ${ }^{1}$, Marie-Christine Woronoff-Lemsi ${ }^{2}$, Mathieu Molimard ${ }^{3}$ et les participants de la Table \\ Ronde $n^{\circ} 7$ de Giens 2009* \\ 1 Laboratoire Roche, Neuilly sur Seine, France \\ 2 Centre Hospitalier Universitaire de Besançon, Besançon, France \\ 3 Centre Hospitalier Universitaire de Bordeaux, Bordeaux, France \\ Texte reçu le 10 février 2010 ; accepté le 26 avril 2010
}

\begin{abstract}
Mots clés : remboursement ; anticancéreux ; survie globale; survie sans progression; critères ;

Commission de Transparence

Résumé - L'évaluation pour l'admission au remboursement faite par la Commission de la Transparence de la Haute Autorité de Santé (HAS) comporte une cotation du Service Médical Rendu (SMR) qui donne l'accès au remboursement, de l'Amélioration du Service Médical Rendu (ASMR) qui détermine la valeur thérapeutique ajoutée et une définition de la population cible. L'évaluation au remboursement des anticancéreux ne diffère pas de celle des autres classes thérapeutiques exceptés certains éléments spécifiques. La survie globale (OS) considérée par la commission de la transparence comme le critère d'évaluation du bénéfice clinique, n'est pas applicable en tant que critère principal dans tous les types de cancer. L'existence de lignes ultérieures de traitement peut rendre difficilement interprétable une OS prise comme critère principal. La survie sans progression (PFS) pour les situations métastatiques et la survie sans maladie (DFS) dans les situations adjuvantes sont des critères tout à fait pertinents pour statuer sur le remboursement d'un nouvel anticancéreux. L'évaluation de la quantité d'effet se fait sur les courbes de survie actuarielles du produit par rapport à son comparateur. L'interprétation des résultats se fait en général sur la médiane de survie qui n'est pas un bon critère puisqu'elle ne mesure qu'un seul point de la courbe. Le hazard ratio mesure l'effet du traitement sur toute la durée du suivi et est ainsi plus global pour quantifier le bénéfice clinique. La détermination d'un seuil de quantité d'effet pour l'octroi du remboursement est difficile, compte tenu de la diversité des situations cliniques des cancers et du niveau de besoin thérapeutique. L'évolutivité rapide des comparateurs ainsi que l'apparition et la recherche en cours de développement de facteurs prédictifs d'efficacité (biomarqueurs, histologie...), peuvent amener à des ASMR différentes selon les populations voire à la restriction de la population cible à un sous groupe de la population de l'Autorisation de Mise sur le Marché (AMM), où la quantité d'effet attendue est plus importante. Pour répondre à ces enjeux, la Table Ronde recommande la possibilité d'avis scientifiques précoces auprès du bureau de la commission de la transparence, pour discuter des comparateurs et de la pertinence de sous groupes de répondeurs. Elle recommande aussi la possibilité d'octroi d'une ASMR temporaire, sous condition de confirmation ultérieure par production de données, lorsque le remboursement semble justifié dans une sous population de l'AMM pour laquelle seule une analyse en sous groupe est disponible.
\end{abstract}

\section{Introduction}

L'évaluation au remboursement est faite par la Commission de la Transparence de la Haute Autorité de Santé (HAS) avec une cotation du Service Médical Rendu (SMR) qui donne l'accès au remboursement. Le SMR tient compte du caractère de gravité de l'affection traitée (morbidité, mortalité), du rapport efficacité/tolérance, de la place du médicament dans la stratégie thérapeutique (notamment par rapport aux autres thérapies disponibles) et de l'intérêt de santé publique (fardeau de la maladie, couverture

\footnotetext{
^ Pour la liste des participants, voir en fin d'article.
}

du besoin thérapeutique et réponse par rapport au besoin, transposabilité des données dans la vie réelle, impact sur le système de soin).

Le SMR est qualifié de majeur, important, modéré, ou faible, donnant accès au remboursement avec des taux allant de $75 \%$ à $15 \%$, ou d'insuffisant pour justifier le remboursement.

La Commission évalue aussi l'Amélioration du Service Médical Rendu (ASMR) qui est la valeur ajoutée relative compte tenu de la taille de l'effet, par rapport à un (des) comparateur(s) et à défaut par rapport à la stratégie thérapeutique. Les critères d'évaluation et des règles méthodologiques définissent un niveau de preuve 
et la quantité d'effet est appréciée non seulement par sa significativité statistique mais aussi par sa pertinence clinique. Enfin, la commission définit la population cible, celle pour laquelle elle recommande le remboursement, cette population pouvant correspondre à un sous groupe de la population de l'indication octroyée dans l'AMM.

Le niveau d'ASMR n'impacte pas directement le remboursement mais le niveau de prix accordé par le Comité Économique.

La problématique de l'évaluation au remboursement des anticancéreux est liée aux critères d'évaluation. En effet, la survie globale [overall survival (OS)], considérée par la commission de la transparence comme le critère d'évaluation du bénéfice clinique, n'est pas applicable en tant que critère principal dans tous les types de cancer, notamment pour les maladies lentement évolutives et lorsque des traitements de rattrapage sont disponibles. L'existence de traitements des lignes ultérieures, surtout lorsqu'ils apparaissent en cours de développement, peut rendre difficilement interprétable une OS prise en critère principal et il n'est pas certain que le bénéfice clinique recherché soit toujours exclusivement fondé sur la survie, certains cancers devenant des maladies chroniques grâce à l'amélioration de leur prise en charge.

Ainsi, hormis la survie globale, quels autres critères sont à considérer pour quantifier le bénéfice clinique en oncologie? De plus, quel que soit le critère retenu, quelle quantité d'effet est considérée comme cliniquement pertinente pour conduire au remboursement? D'autres questions interviennent également pour étayer la problématique, comme la prise en compte du besoin thérapeutique dans l'appréciation de la quantité d'effet ou encore le choix des comparateurs pertinents à retenir compte tenu de l'évolutivité des stratégies thérapeutiques et la population cible.

\section{Méthodologie}

Pour répondre à la question posée, la table ronde s'est d'abord attachée à passer en revue les critères d'efficacité actuellement pris en compte en oncologie, selon les recommandations de l'European Medicine Agency (EMA) et de la Food and Drug Administration (FDA) disponibles. ${ }^{[1,2]}$

Une revue de la littérature a également été effectuée, notamment sur l'analyse de la variabilité de ces critères et leur validité dans les différents types de cancers. Enfin, une analyse des avis de la commission de la transparence sur des anticancéreux, émis entre début 2007 et juin 2009, a été réalisée pour tenter d'identifier les critères pris en compte et la méthodologie choisie pour estimer la quantité d'effet.

Dans un deuxième temps, sur la base des recherches effectuées, des tableaux croisés pour chaque critère d'efficacité ont été établis, afin de hiérarchiser leur pertinence selon le type de cancers. La revue d'autres critères que les critères d'efficacité a aussi été réalisée afin de déterminer la pertinence de leur prise en compte dans l'avis de la transparence. Dans ce cadre, la question de la disponibilité des données au moment de l'évaluation au remboursement a été envisagée de manière à définir les éléments à produire dans des études complémentaires après mise sur le marché.

\section{Les critères pertinents pour le remboursement des anticancéreux}

\section{1. Les critères d'efficacité}

En oncologie, l'OS est le critère dont la pertinence clinique est maximale puisqu'il correspond au premier bénéfice clinique que l'on souhaite apporter aux patients et il s'agit d'une mesure sans biais d'évaluation : c'est LA mesure d'efficacité reconnue par la Commission de la Transparence. Elle est définie par le délai entre la randomisation et le décès quelque soit la cause, ou la date de dernière nouvelle. L'écueil réside dans la nécessité, pour certains cancers où les décès sont rares, d'un suivi prolongé et d'un grand nombre de patients à inclure. En cas de survie prolongée, les décès non liés au cancer viennent souvent diluer l'effet, rendant difficile l'appréciation de l'effet du traitement dans le cadre de risques compétitifs d'autres causes de décès dont l'âge. Enfin, pour les traitements de première ligne, la disponibilité de traitements de lignes ultérieures, les essais croisés (cross over) et les changements de traitement (switchs) sont aussi des facteurs confondants pour l'interprétation de la survie globale. ${ }^{[3-5]}$

De nombreux autres critères d'efficacité sont évalués dans les essais et des recommandations sont disponibles pour décrire et évaluer l'activité anti-tumorale d'un traitement. ${ }^{[6,7]}$

La survie sans maladie [Disease Free Survival (DFS)], dans les situations adjuvantes, et la survie sans progression [Progression Free Survival (PFS)] pour les situations métastatiques, sont considérés comme des critères de substitution de l'OS dans certains cancers pour lesquels une corrélation a été validée (cancer du colon). ${ }^{[8-11]}$ Dans les cas non validés comme le cancer du sein, la PFS est considérée comme un critère intermédiaire. ${ }^{[12,13]}$ La DFS est définie par le délai entre la randomisation et la récidive locale ou à distance. La PFS est définie par le délai entre la randomisation et la progression, un second cancer ou le décès quelle que soit la cause. Ces critères ont une pertinence clinique indéniable puisqu'ils mesurent le temps durant lequel il n'y a pas de manifestation clinique d'une progression tumorale, ils reflètent ainsi plus directement l'action anti-tumorale que la survie et ne sont pas influencés par les lignes ultérieures de traitement. L'écueil de ces critères réside dans le biais du moment de la mesure, puisque la 
progression est le plus souvent évaluée au temps prévu par le protocole et non pas au temps de la progression elle-même. L'évolution des connaissances et des technologies influence la méthode de mesure de la progression et prive l'évaluateur de l'historique des valeurs de référence. Enfin, sur le plan biométrique, une deuxième lecture par une revue indépendante ou une lecture centralisée améliore les spécificité, sensibilité et reproductibilité de la mesure sans en éliminer les biais. ${ }^{[14-17]}$

La réponse tumorale ou biologique définie par le taux de réponse est un critère explicatif documentant l'action directe des traitements sur la masse tumorale. Il s'agit du taux de réponse objective [Objective Response Rate (ORR)] et du taux de réponse complète [Complete Response Rate (CRR)] considérés le plus souvent comme des critères intermédiaires, sauf dans les hémopathies malignes où ils sont assimilés à des critères de substitution. Bon outil dans le suivi clinique usuel des patients, ils peuvent être évalués précocement par rapport à la survie ou à la progression et sont utiles dans les phases précoces de développement ou en l'absence de randomisation.

\subsection{Les autres critères}

La tolérance est prise en compte par la Commission de la Transparence pour venir pondérer l'estimation de l'efficacité et juger ainsi la valeur thérapeutique. Quand la quantité d'effet est faible, la fréquence et l'intensité des effets indésirables sont pris en compte pour qualifier la qualité du gain de survie. La Commission déplore souvent l'absence de données de qualité de vie : les outils existent en oncologie mais leur utilisation répétée dans les essais cliniques est difficile et leur variabilité interindividuelle est grande, ce qui diminue leur pertinence pour discriminer des traitements ou des stratégies thérapeutiques.

$\mathrm{Du}$ fait de la chronicisation des cancers, d'autres outils comme l'évaluation de la préférence des patients ou de l'utilité pourraient être pris en compte par la Commission de la Transparence en oncologie.

\section{Appréciation de la quantité d'effet}

La notion de quantité d'effet pour le SMR et l'ASMR intègre l'effet (sur quoi et quel critère) et la quantité (combien). Son appréciation est différente de celle réalisée en AMM, la Commission recherchant particulièrement la pertinence clinique d'une quantité d'effet statistiquement significative en tenant compte du besoin thérapeutique, du fardeau de la maladie, de la population cible et surtout de la capacité à extrapoler (transposer) les résultats des essais à la pratique réelle. La nature comparative des données est un élément indispensable à l'appréciation de la quantité d'effet.

\section{1. La médiane de survie}

Le critère principal que ce soit l'OS, la PFS ou la DFS s'exprime sous la forme de courbes de «survie» actuarielles ou de Kaplan-Meier. L'estimation de la quantité d'effet par la Commission se base le plus souvent sur la médiane de survie qui est l'estimation de la survie de la population à un temps $t$. Cette expression de la quantité d'effet est un critère facilement compréhensible mais elle ne représente qu'un seul point de la courbe de survie et dans certains cas peut ne pas être atteinte dans un bras au moment de l'évaluation.

\subsection{Le rapport des hasards ("hazard ratio »)}

L'autre expression de la quantité d'effet est le « hazard ratio ». C'est le rapport des risques instantanés d'événements entre le bras A (bras expérimental) et le bras B (bras contrôle).

$$
H R=\frac{\text { risque de décès dans } \mathrm{A}}{\text { risque de décès dans } \mathrm{B}} \text {. }
$$

Dans un essai randomisé, c'est aussi le rapport des incidences approché par

$$
H R=\frac{(\text { nb décès } d s A / \text { suivi total })_{\text {bras } \mathrm{A}}}{(\text { nb décès } d s B / \text { suivi total })_{\text {bras } \mathrm{B}}} .
$$

Si HR > 1 alors le risque sous A est supérieur à celui sous B et on conclut à l'effet nocif de A.

$\mathrm{Si} \mathrm{HR}=1$ alors le risque sous $\mathrm{A}$ est égal à celui sous B et on conclut à l'absence d'effet de A.

$\mathrm{Si} \mathrm{HR}<1$ alors le risque sous $\mathrm{A}$ est inférieur à celui sous $\mathrm{B}$ et on conclut à l'effet bénéfique de $\mathrm{A}$.

Cette mesure exprime une valeur relative, à l'inverse de la médiane de survie qui est une valeur absolue. Son interprétation nécessite donc la prise en compte du risque dans le bras comparateur et repose sur l'hypothèse de proportionnalité des risques. L'intérêt majeur du hazard ratio est qu'il mesure l'effet du traitement sur toute la durée du suivi.

Ainsi, pour une même différence de médiane de survie, le « hazard ratio » peut révéler des écarts majeurs permettant la différentiation de deux produits et est donc complémentaire.

\subsection{Le comparateur}

L'évaluation de l'effet du traitement dépend de la nature comparative de l'essai et de la pertinence du traitement de comparaison, cette pertinence étant jugée selon la population de l'essai. Le comparateur peut être un médicament ou une stratégie de prise en charge reconnue. Si le comparateur choisi n'est pas reconnu 


\title{
Prise en compte du besoin thérapeutique dans l'appréciation de la quantité d'effet
}

\author{
Incidence et mortalité des différents cancers en 2005
}

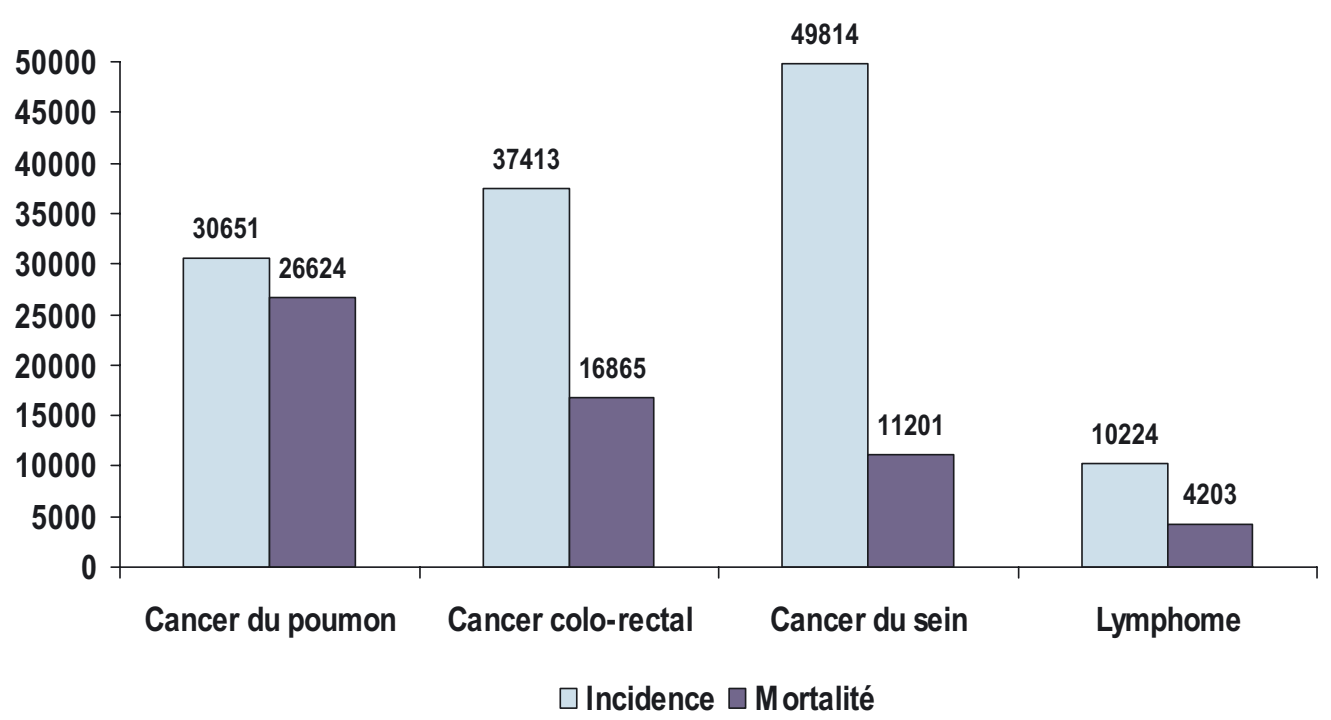

Source : Données INVS

Fig. 1. Prise en compte du besoin thérapeutique dans l'appréciation de la quantité d'effet.

comme un des meilleurs par la Commission de la Transparence, il y a un risque de dégradation de l'ASMR voire du SMR. Or la difficulté en oncologie pour juger de la pertinence du comparateur réside en la rapidité de changement de stratégie thérapeutique. Le recours à des avis d'experts prend toute son importance dans ce cadre. Pour les autres aspects du choix du comparateur, la Table Ronde propose de se référer aux recommandations de la Table Ronde $\mathrm{n}^{\circ} 1$ : Les comparateurs (médicamenteux et non médicamenteux) pour l'AMM, pour la Santé Publique, pour les payeurs et au niveau Européen.

\subsection{La population cible}

La population cible retenue pour le remboursement est en règle générale la population de l'AMM. Cependant, en oncologie comme dans d'autres domaines thérapeutiques, elle peut être restreinte par rapport à la population de l'AMM. Dans d'autres cas, la Commission de la Transparence peut définir des sous-populations avec des ASMR de niveaux différents. L'évaluation de la quantité d'effet dans ces sous-populations se fait sur la base d'analyses en sous groupes, sous réserve que ces analyses aient été prévues dans le protocole. Cependant, l'évolution des données scientifiques, notamment l'identification de biomarqueurs au cours du développement, devrait amener la commission à considérer des données d'efficacité provenant d'analyses post hoc, non initialement prévues par le protocole. Pour être recevables, ces analyses doivent être réalisées avec toute la rigueur méthodologique nécessaire, en réalisant notamment un test d'interaction et la Commission pourrait, pour en vérifier la rigueur, être amenée à demander la mise à disposition des données individuelles. Une autre possibilité évoquée par le groupe de travail pour des thérapies «ciblées » dont les données ne sont pas «matures » dans une sous population par exemple, serait l'octroi d'une ASMR temporaire sous condition de confirmation par une évaluation ad hoc.

Pour les autres aspects de la population cible, la Table Ronde propose de se référer aux recommandations de la Table Ronde $\mathrm{n}^{\circ} 5$ : Définition des populations cibles : comment anticiper au mieux la définition des populations cibles en vue du remboursement : sources de données, raisonnement...

\subsection{Le besoin thérapeutique}

Le besoin thérapeutique en oncologie ne peut être abordé de manière univoque, le cancer recouvrant une réalité clinique et des approches thérapeutiques très diverses. Une manière d'aborder le besoin thérapeutique est de rapprocher l'incidence et la mortalité de 4 cancers fréquents (figure 1). Une même quantité absolue d'effet (gain de survie de 2 mois par exemple) doit-elle être appréciée 
de la même manière dans le cancer du sein et du poumon? De la même façon, une même quantité de réduction du risque relatif dans le cancer du sein et du poumon doit-elle être appréciée de la même manière?

La traduction de la quantité d'effet en ASMR tiendra compte de la valeur de base du critère, la survie moyenne pour la pathologie concernée étant prise en compte pour apprécier la valeur ajoutée thérapeutique. Ainsi l'existence d'un besoin thérapeutique important ou non couvert impacte l'évaluation au remboursement des traitements en général et des anticancéreux en particulier.

\section{Conclusion et recommandations}

La Commission de la Transparence dans son évaluation du remboursement des anticancéreux requerrait des données de survie globale. Or la survie globale (OS) n'est pas toujours le critère principal idéal. Ainsi la survie sans progression (PFS) pour les situations métastatiques ou la survie sans maladie (DFS) dans les situations adjuvantes, sont des critères tout à fait pertinents dans les maladies lentement évolutives ou quand les traitements de rattrapage sont disponibles. Toutefois, dans ces lignes précoces de traitement, et dans la mesure où l'objectif thérapeutique ultime d'un anticancéreux est de prolonger la survie, en cas de choix de la PFS comme critère principal, l'OS doit toujours être étudiée comme critère secondaire et le suivi de la survie assuré. Son interprétation peut souvent soulever des questions notamment lorsque, pour des raisons éthiques évidentes, des traitements de deuxième ligne ont été administrés. Quant au taux de réponse seul, il n'est pas accepté par la Commission de la Transparence comme un critère d'évaluation pour le remboursement excepté dans le cas particulier des hémopathies malignes.

L'évaluation de la quantité d'effet se fait sur les courbes de survie actuarielles du produit par rapport à son comparateur. L'analyse de cette information se fait souvent sur la médiane de survie, mais en l'absence de paramètre idéal, la recommandation est de faire appel à plusieurs paramètres : le rapport des hasards (hasard ratio) avec son intervalle de confiance (IC), la médiane de survie globale ou de survie sans progression ainsi que le taux de survie à un temps $t$.

Hormis les critères décrits ci-dessus, l'évaluation pour l'admission au remboursement des médicaments anticancéreux ne diffère pas de celle des autres classes thérapeutiques. La tentative par le groupe de travail de déterminer un seuil de quantité d'effet pour l'octroi du remboursement n'a pu aboutir, puisque ce seuil dépendrait du besoin thérapeutique, du comparateur et de l'évolution des connaissances dans le domaine concerné.

Pour autant l'évolution rapide des stratégies thérapeutiques de référence, liée à la richesse des portefeuilles de produits
(700 molécules en développement) d'une part et la découverte en cours de développement de facteurs prédictifs d'efficacité (biomarqueurs ou histologie) d'autre part, ont amené la Table Ronde à faire deux recommandations. La première est la possibilité pour l'industrie pharmaceutique d'obtenir des réunions d'échange avec des avis scientifiques précoces auprès du bureau de la Commission de la Transparence. Les discussions porteraient essentiellement sur le choix des comparateurs et la pertinence de sous groupes de répondeurs, notamment liés à l'identification de nouveaux facteurs prédictifs de réponse. Cet échange peut amener à une évaluation différente de l'ASMR selon les populations voire à un SMR différent. Il est possible aussi qu'en cas de quantité d'effets jugée insuffisante dans la population de l'AMM que le remboursement soit envisagé avec une la restriction de la population cible à un sous groupe où la quantité d'effet attendue est cliniquement pertinente. La deuxième recommandation est, en cas d' «immaturité » de certains résultats d'efficacité, la possibilité d'octroi d'avis de transparence temporaires, avec une ASMR temporaire, sous condition de confirmation.

Participants. Jean-Pierre Armand (Institut Claudius Regaud, Toulouse), Marc Bardou (CIC, Inserm, Dijon), Loïc Bergougnoux (Laboratoire Roche), Christine Bouchet (Laboratoire Novartis), Dominic Cellier (Laboratoire Merck), Bachir Dahmani (Haute Autorité de Santé, Saint-Denis), Bertrand Diquet (CHU, Angers), Thierry Lecomte (CHU, Tours), Nathalie Hoog Labouret (InCa, Boulogne Billancourt), Claire Labreveux (Laboratoire GlaxoSmithKline), François Meyer (Haute Autorité de Santé, Saint-Denis), Gilles Paintaud (CHRU, Tours), Pascal Piedbois (Laboratoire Astra-Zeneca), Martine Pigeon (Cnamts, Paris), Olivier Amédée Manesme (Paris Biotech, Paris), Rémy Defrance (Laboratoire Pfizer), Dominique Tonelli (Laboratoire Bristol Myers Squibb), Karen Pinachyan (Laboratoire Murigenetics), Sophie Tardieu (AP-HM, Marseille).

\section{Références}

1. EMEA. Guideline on the evaluation of anticancer medicinal products in man (CPMP/EWP/205/95 Rev.3/Corr.2, Dec 2005) http://www . ema. europa eu/pdfs/human/ewp/020595en.pdf

2. FDA. Guidance for industry, clinical trial endpoints for the approval of cancer drugs and biologics (May 2007) http://www.fda.gov/downloads/ Drugs/GuidanceComplianceRegulatoryInformation/Guidances/ ucm071590.pdf

3. Mathoulin-Pelissier S, Gourgou-Bourgade S, Bonnetain F, et al. Survival end point reporting in randomized cancer clinical trials: a review of major journals. J Clin Oncol 2008; 26: 3721-6

4. Pazdur R. Endpoints for assessing durg activity in clinical trials. Oncologist 2008; 13(supp12): 19-21

5. Pignatti F, Aronso B, Vamvakas S, et al. Clinical trials for registration in the European Union: the EMEA 5-year experience in oncology. Critical Reviews in Oncology/Hematology 2002; 42: 123-35 
6. Eisenhauer A, Therasse P, Bogaerts J, et al. New response evaluation criteria in solid tumours: revised REGIST guideline (version 1.1). European Journal of Cancer 2009; 45: 228-47

7. Cheson B, Pfistner B, Juweid ME, et al. Revised response criteria for malignat lymphoma. J Clin Oncol 25: 579-86

8. Buyse M , Burzykowski T, Caroll K, et al. Progression-free survual is a surrogate for survival in advanced colorectal cancer. J Clin Oncol 2007; 25: 5218-24

9. Punt CJ, Buyse $\mathrm{M}$, Köhne $\mathrm{CH}$, et al. Endpoints in adjuvant treatment trials: a systematic review of the literature in colon cancer and proposed definitions for future trials. J Natl Cancer Inst 2007; 99: 998-1003

10. Sargent D, Wieand H, Haller D, et al. Disease-free survival versus overall survival as a primary end point for adjuvant colon cancer studies: individual patient data from 20,898 patients on 18 randomized trials. J Clin Oncol 2005; 23: $8664-70$

11. Tang P, Bentzen S, Chen E, et al. Surrogate end points for median overall survival in metastatic colorectal cancer: literature-based analysis from 39 randomized controlled trials of first-line chemotherapy. J Clin Oncol 2007; 25: 4562-8

12. Hudis C, Barlow W, Costantino J, et al. Proposal for standardized definitions for efficacy end points in adjuvant breast cancer trials: The STEEP system. J Clin Oncol 2007; 25: 2127-32
13. Miksad R, Zietemann V, Gothe R, et al. Progression-free survival as a surrogate endpoint in advanced breast cancer. Int $\mathrm{J}$ of Technol Assess Health Care 2008; 24 (4): 371-83

14. Knox J. Progression-free survival as endpoint in metastatic RCC? Comment $\mathrm{n}^{\circ} 559157$ The Lancet Vol 372 August 9, 2008

15. Freidlin B, Korn E, Hunsberger S, et al. Proposal for the use of progressionfree survival in unblinded randomized trial. J Clin Oncol 2007; 25: 2122-6

16. Fleming $\mathrm{T}$, Rothman $\mathrm{M}$ and $\mathrm{Lu} \mathrm{H}$. Issues in using progression-free survival when evaluating oncology product. J Clin Oncol 2009; 27: 2874-80

17. Dodd L, Freidlin B, Jaffe C, et al. Blinded independent central review of progression-free srvival in Phase III clinical trials: important design element or unnecessary expense? J Clin Oncol 2008; 26: 3791-6

Correspondance et offprints : Rima de Sahb-Berkovitch, Affaires Économiques, Laboratoire Roche, 52 boulevard du Parc, 92521 Neuilly sur Seine Cedex, France.

E-mail : rima.de_sahb-berkovitch@roche.com 